\title{
Analysis and Design of Radio and TV NGB Construction Model
}

\author{
Peifeng Li
}

Jiangsu Province Xuzhou Technician Institute, Xuzhou, Jiangsu, 221000

Keywords: NGB; model; design

\begin{abstract}
By analyzing the current network technology system, development trend and development status of radio and television, the article clearly defined the opportunities and challenges of radio and television in the context of NGB and designed the NGB construction model of radio and television. Unidirectional broadcast service is the most basic business of radio and television NGB model currently the largest proportion of the basic type of digital TV set-top boxes, but are reserved for two-way function, you can add a cable modem via EPON or way into two-way set-top boxes, Two-way business is the most important part of the development of radio and television NGB model, change the traditional viewing habits are mainly two-way on-demand television, the real advantage of digital television is on-demand television, rather than just digital after adding a few units. Integration and exchange of radio and television broadcasting is the NGB's advanced goal of building a truly full-service cross-network integration and interoperability in order to better play the advantages of radio and television to bring better social and economic benefits.
\end{abstract}

\section{Introduction}

In recent years, China has witnessed rapid development in economy, culture, science and technology. In the new market environment of triple play, both China Mobile, China Telecom, China Unicom and Chinese radio and television operators are faced with the adjustment of development strategy and market positioning The issue of competition among major operators is becoming increasingly fierce. In order to meet people's growing material and cultural needs, comply with the historical development trend of triple play, and at the same time enhance the business carrying and supporting capacity of the radio and television NGB model in the face of fierce market competition, SVA set out in early 2009 to build enough to carry interactive video on demand, Broadband Internet access, MPLS VPN leased line, IP-Phone and streaming media and other value-added services covering the city's next-generation data network platform.

Radio NGB data network platform to meet the advanced construction, economy, high availability, scalability, security and manageability and other general network design requirements, and to meet the radio and television NGB model broadband IP network as the latest carrier-class broadband IP network Required reliability, QoS, scalability, network connectivity, communication protocols, network management and security, multicast and other requirements.

Cisco7609 router as the core switching node to provide penetration services for high-speed data forwarding, providing high-speed city IP data exit and high-performance firewall, responsible for the various convergence layer link up, carrying interactive services, broadband services, and VPN data traffic Fast forwarding, and broadband billing system access.

Accurate access to broadband billing system for the protection of broadband revenue economy, safeguarding the legitimate rights of broadband users is essential. Generally speaking, the broadband charging system should be connected in series between the egress router and the core router to ensure that all users must be verified by the broadband charging system before they can access the extranet. After the valid user enters the correct account and password, Smooth access to the Internet.

In the CMTS + CM access mode, the CM needs to go through the nine-step interaction with the CMTS to go online normally. Failure of any of these steps will result in the CM not being able to go online. Users' set-top boxes and PCs can not obtain legal IP addresses from DHCP, thus preventing interactive and broadband services. In order to quickly diagnose and troubleshoot problems, Radio 
and TV NGB model will be dedicated to CMTS and CM interaction process and the significance of each step possible causes of failure into a book, etc., in the event of a similar failure can be quickly compared to greatly improve the fault judgment The accuracy thus reduces the time it takes to troubleshoot.

The new generation of radio and television data network platform is facing 300,000 radio and television users, it is necessary to IP for an efficient and practical address planning. And uses some routing protocol algorithms, such as OSPF, MPLS, etc. [10] to realize the efficient operation of the network through the use of advanced algorithms and to provide a technical support platform for the development of new services of broadcasting and TV data.

\section{CMTS network management system analysis and design}

For a variety of network equipment, in order to facilitate the monitoring of its performance, and timely understanding of its work, can be set up in the engine room network management system software, monitoring equipment traffic and bandwidth management, network performance monitoring and network identification and network error management and CMTS current letter Noise ratio, level, the number of CM.

In order to ensure the real-time monitoring of CMTS operation of the NGB model of radio and television and judge the current running status of the entire system, the CMTS network management system is installed in the radio and television network center.

The system can monitor, analyze and early warning the main network technical parameters, which can help to improve the operation and maintenance efficiency and reduce the pressure for maintenance of personnel.

According to the actual usage of the light platform of the current broadcasting and broadcasting as follows, the NGB model of radio and television designs a new architecture of the optical platform network management:

1) Although the Motorola optical platform does not meet the national standard, the radio NGB model already has its mib library, and its NMS has been used normally for many years, so the secondary development of its integration is relatively simple. And Conte, Lei Huaguang platform in line with the national standard. The remote management interfaces of the three optical platforms are based on the IP RJ45 network interface;

2) Radio and TV NGB models need to manage the optical platforms are indoors;

3) SVA has now built a central room - sub-front-end monitoring room network switches in addition to the trunk port, the other access ports through the VLAN division of the corresponding business. Among them, MOTO equipment network planning VLAN20, the port is 5 16 \#, up to 12 devices. Radio and TV NGB model Conte, Lei Huaguang platform network management interface is also planned into the VLAN20, so that you can smooth the MOTO network management radio and television NGB model construction of the new network management. For the front end with a large amount of optical platform, the port reserved for VLAN 60 is assigned to VLAN20.

Radio NGB NMS model design architecture as shown below. NMS architecture for the distributed management system, according to the center room - sub-front-end layout, sub-front-end can run independently, but its management information in the central engine room summary. In actual use, in order to save the cost, the NGB model of Radio and TV sets the network management database and the network management software server and the network management client to be installed on the same server.

\section{Radio and television NGB model of a new generation of terminal platform analysis and design}

Radio and television do not agree to the level of radio and television front-end system as a measure of the level of development of radio and television networks the only indicator of the "radio and television network digital, interactive success" conclusion. Radio and television network transformation if there is no terminal support, how to achieve triple play? How to conduct business? 
How to meet the ever-increasing cultural needs of the broad masses of people? How to deal with the fierce competition from other telecom operators? - Almost all value-added services, including interactive video on demand, broadband Internet access, MPLS VPN leased line, IP-Phone and streaming media, all rely on the support of the terminal platform.

Some radio and television talked about technology, talked about innovation, that is, come up with their own how to build a new generation of radio and television front-end platform in an example, very curious: no terminal platform, what to receive? Terminal platform also has the technology of gold, but also innovation; not optional, unrelated to the overall situation. However, if the broadcasting network does not mention the terminal, as a user will take it for granted that digital television is a cable television, radio and television network is just radio and television, not the Internet.

Since the broadcast NGB model will highlight this chapter and introduce it as a big chapter, then the importance of the terminal platform and the great efforts of the broadcasting NGB model on the terminal platform will be explained. Radio and television NGB model through the innovative platform for terminal construction, to bring prosperity to radio and television, in order to meet the spirit of the general public, material and cultural needs to make outstanding contributions.

The construction of a new generation of broadcasting NGB model terminal platform to meet the advanced, economical, high availability, scalability, security and manageability and other design requirements, network culture on human survival and social evolution have a full range of impact. Due to the ubiquity, transnationality, complexity and vulnerability of the Internet, the protection of national information security, cultural security and social stability are the top priorities [13]. At the same time, it meets the requirements of the wideband IP network of NGB model of radio and television as the reliability, QoS, scalability, network interconnection, communication protocol, network management, security and multicast required by the latest operational level broadband IP network.

The new generation terminal platform of NGB model of radio and television is in charge of such systems as EPG information release and advertising system, IP-based multicast service system, set-top box network management system, broadband authentication system, data broadcasting system, stock system, CMTS system and digital television system Docking the task; at the same time docking radio and television departments, involving the radio and television network transmission center of the front-end platform docking, business development department of the new business docking, operation and maintenance of the terminal troubleshooting, the Chief Engineer and Engineering Department of the network design transformation.

In addition, several important technological innovations of the NGB model include: flexible and diversified EPG information dissemination and advertising system after digitization; different from traditional PPPoE, DHCP, terminal pre-authentication redistribution IP address technology and multicast technology [ 14]; radio and television NGB model in the distinction between business and laid the foundation for the development of new business, based on the DHCP + creative use of the international RFC standard OPTION 60 technology; authority level bidirectional network failure on-site inspection system in the terminal In the new video mode, the NGB model of radio and television considered the transmission of 3D TV in the cable digital television system in advance. In addition, and for this, the set-top boxes ahead of planning, are NTSC terminal platform for radio and television, and even the big innovation in the broadcasting system sector innovation point.

Radio and TV NGB model built to support the launch of the boot screen / video, shutdown screen, channel switching, embedded audio / video pages, audio, data broadcasting, NVOD, stock system, rolling subtitles, push screen, Application of advertising carrier platform - a new generation of EPG information dissemination and advertising system [15].

The new generation EPG information broadcasting and advertising system of the NGB model of radio and television supports the arrangement of advertisement data in the form of audio and video, pictures and texts, the flexibility of selecting broadcast positions and broadcasting time, different broadcasting strategies and the like, and these functions are the broadcast NGB model The future of flexible information dissemination and advertising strategies to provide support. 
With the development of interactive services, providing a platform for opening up an open network with the network. The loose coupling with the network can make the service independent of specific access technologies and facilitate the independent development of the service itself [16]. Based on the technologies such as IP-QAM already supported in the terminal system, the NGB model of broadcasting needs some new advantages of IPTV set-top boxes, such as VLAN functions, IGMP snooping v1 / v2 support, IGMP proxy support, Support the transparent transmission of EAPOL, can group the set-top box and CPE that it brings, as the member of the multicast. You can view the set-top box in the multicast table and the multicast address that the CPE that it joins.)

\section{4. $3 \mathrm{D}$ high-definition digital television system design}

Through the cable TV can make the audience more convenient to share 3D video, but the 3D video business data volume, its bandwidth saving has high requirements, so the efficiency of data compression directly affects the 3D video services market applications, and compression The premise is not to seriously affect the audience's perception of 3D visual quality.

The depth of the human eye is the sense of three-dimensional because the left and right eye parallax, and then formed by the brain processing, so the most basic 3D network video architecture is based on two perspectives of multi-perspective system, left and right two-way video through 3D The final display after decoding. However, with the development of 3D technology, the architecture also gradually changes.

At present, the video in the cable is all $2 \mathrm{D}$, and the receiving receiver box of the terminal only supports 2D. Therefore, when using 3D, it is also necessary to consider the support for 2D, as shown in Figure 3-16. But when using different compression encoding system is different.

$2 \mathrm{D}$ and $3 \mathrm{D}$ independent recording, are converted into 2D, 3D two views, and then separately encoded and transmitted through the digital television network, respectively, in the terminal to decode and display.

There are many existing compression standards for normalization, but different compression standards adopt very different techniques and their coding models are different. See Table 3-1 for the compressed modeling and coding. A good model has a high compression rate, good error resistance, but the general complexity will be higher.

At present, the coding efficiency of several models based on motion region, object, face, and sound image description is relatively high, but at present the more widely used is H.263 + (it is still to be noticed that H.264 and H.265), MPEG4, AVS, MPEG-4 generally used for storage and playback, streaming media broadcast or H.264 or AVS-based, mature and stable coding standard should be adopted in China's cable TV system, H.264 or AVS can be used. Support for H.264 devices is widely used, but involves intellectual property; AVS is our own intellectual property rights, but the problem is that support for this standard is not a wide range of H.264 vendors.

MVD achieves a higher quality visual experience but requires more hardware. The quality of the output view depends on the number of encoded videos, the compactness of the delivery, the accuracy of the depth information, and the quality of the view differences, in front of MVD technology is not yet mature [17], the main face of depth estimation, depth coding, view synthesis and other technical challenges.

On the basis of the channel coding of the traditional 2D digital television, the 3D QOS must be considered. The basic idea is to allocate a higher bit rate to the important information codes in the $3 \mathrm{D}$ video, and to give higher priority to transmission during transmission. protection. For example, during $\mathrm{V}+\mathrm{D}$ video transmission, the priority of the color image should be higher than the priority of the depth information to ensure that the user's video viewing is performed under bad channel conditions.

Initially, considering the market conditions and cost factors, it is not advisable to use the encoding of the depth information because the terminal set-top box of the 2D digital television can be used directly to deploy $3 \mathrm{D}$ at the front based on the conventional 2D cabled digital television system (EPG, CA) Multi-view video encoding equipment (such as AVS MVC encoding), and then into the multiplexing equipment, and QAM256 modulation, transmission through the cable HFC 
network, in the user's home, with integrated 3D decoder STB, the last access to stereoscopic display devices, To achieve 3D viewing, see Figure 3-20. To mid-term can use V + D, MVD coding method.

In cable TV, 3D can be compatible with traditional 2D TV. The use of 3DTV in existing cable TV systems can be realized through the selection of good modeling and compression methods and source coding methods. However, the transmission of 3DTV in cable TV systems Faced with many problems to be solved.

\section{Acknowledgement}

Jiangsu Provincial Institute of Vocational and Technical Education project (XHXS2017066)

China Association of Staff Education and Vocational Training 2017 key research topics (2017004)

\section{References}

[1] Wang Liang. NGB Intelligent Terminal Solution for Modern Information Service [J]. Technology \& Television Technology, 2011, (4): 7-9.

[2] HUA K A, CAI Y, SHEU S. Patching: a multicast technique for true video-on-demand services [C]. Proceedings of ACM Multimedia'98.1998.191-200.

[3] Li Lei. Application of RADIUS in Next Generation Broadcasting Network (NGB) [J]. Cable Television Technology, 2011, (2): 55-59.

[4] YANG Pi-Jin. Using DOCSIS to Upgrade and Upgrade NGB Access Network [J]. Journal of Radio and Television, 2011, (3): 102-104. 